\title{
Comparative Lipidomics of Different Yeast Species Associated to Drosophila suzukii
}

\author{
Flavia Bianchi ${ }^{1,2}$, Urban Spitaler ${ }^{3,4}{ }^{\circledR}$, Peter Robatscher ${ }^{1}{ }^{(0}$, Rudi F. Vogel ${ }^{2}$, Silvia Schmidt ${ }^{3}$ \\ and Daniela Eisenstecken $2, * \mathbb{D}$ \\ 1 Laboratory for Flavours and Metabolites, Institute for Agricultural Chemistry and Food Quality, \\ Laimburg Research Centre, Ora (BZ), 39040 Auer, Italy; flavia.bianchi@laimburg.it (F.B.); \\ peter.robatscher@laimburg.it (P.R.) \\ 2 Chair of Technical Microbiology, School of Life Sciences Weihenstephan, Technical University of Munich, \\ Gregor-Mendel-Straße 4, 85354 Freising, Germany; rudi.vogel@wzw.tum.de \\ 3 Entomology Group, Institute for Plant Health, Laimburg Research Centre, Ora (BZ), 39040 Auer, Italy; \\ urban.spitaler@laimburg.it (U.S.); silvia.schmidt@laimburg.it (S.S.) \\ 4 Institute of Plant Protection, Department of Crop Sciences, University of Natural Resources and Life \\ Sciences, Gregor-Mendel-Straße 33, 1180 Vienna, Austria \\ * Correspondence: daniela.eisenstecken@laimburg.it; Tel.: +39-0471-414846
}

Received: 6 July 2020; Accepted: 26 August 2020; Published: 28 August 2020

\begin{abstract}
Yeasts constitute a dietary source for the spotted wing drosophila (SWD) and produce compounds that attract these flies. The study of the chemical composition of the yeast communities associated with SWD should therefore help to understand the relationship between the biology of the insect and the yeast's metabolism. In the present study, the lipidome of five yeast species isolated from grapes infested by SWD (three Hanseniaspora uvarum strains, Candida sp., Issatchenkia terricola, Metschnikowia pulcherrima and Saccharomycopsis vini) and a laboratory strain of Saccharomyces cerevisiae was explored using an untargeted approach. Additionally, the lipid profile of two species, S. cerevisiae and $H$. uvarum, which were reported to elicit different responses on SWD flies based on feeding and behavioral trials, was compared with a chemical enrichment approach. Overall, 171 lipids were annotated. The yeast species could be distinguished from each other based on their lipid profile, except for the three strains of $H$. uvarum, which were very similar to each other. The chemical enrichment analysis emphasized diversities between S. cerevisiae and $H$. uvarum, that could not be detected based on their global lipid profile. The information concerning differences between species in their lipidome may be of interest to future entomological studies concerning the yeast-insect interaction and could help to explain the responses of SWD to diverse yeast species.
\end{abstract}

Keywords: lipidomic profile; yeast strains; spotted wing drosophila; untargeted LC-MS

\section{Introduction}

Drosophila suzukii Matsumura, the spotted wing drosophila (SWD), is an insect pest which causes severe economic losses and agricultural damages in Italy as well as in other countries worldwide [1-4]. Different yeast species present on fruits are found in association with SWD [5-7], and due to their attractiveness and feeding stimulant activity towards SWD flies, these microorganisms can be employed in baits or attract-and-kill formulations against SWD [8-11]. In fact, yeasts constitute a food source for Drosophila flies [12] as they provide protein, amino acids, lipids, and vitamins to the insect [13-17]. In addition, yeasts can stimulate appetite behavior in SWD flies [7] and encourage insects to feed on yeast-laden food, because they associate it with a sugar source [18]. Yeasts also produce volatile compounds that are attractive to SWD flies $[5,9,19,20]$. Not only the yeast species, but also the strain and growth medium affect the attractiveness [20]. 
The nutritional value of a food source for Drosophila melanogaster is influenced by the protein-tocarbohydrates-ratio [21], and vitamins provided by symbiotic microorganisms are essential food components in the Drosophila diet [12,22]. The same 10 amino acids which are essential to other eukaryotes are also necessary for Drosophila [23], but the requirement of other compounds like proteins, carbohydrates, and lipids are different among Drosophila species, and their availability influences the fitness and life-history traits of the insect $[24,25]$.

Although Drosophila is able to synthetize the necessary fatty acids for its survival [14], little is known about the preferences and nutritional behavior of Drosophila fed with different lipid-containing diets. Carvalho et al. [14] demonstrated that the dietary fatty acids' composition has an effect on the phospholipid compounds present in cell membranes of Drosophila melanogaster. In contrast, the abundance of specific phospholipid classes in various tissues does not reflect the phospholipidic composition of their diet [14]. Lipids are ubiquitous compounds with a key role in numerous biological processes: they are (i) constituents of cell membranes, (ii) involved in signaling pathways, and (iii) a storage energy source [26]. The lipid composition of eukaryotic organisms is influenced by the carbon source and the growth medium composition [27,28]. Therefore, differences in the lipid profile are reflected in the phenotype in specific environmental conditions [29]. Yeasts constitute a lipid source for Drosophila and their lipidome is representative for numerous compound classes. Triacylglycerols (TG) and steryl esters (SE) are reported as the two major nonpolar lipids of the yeast S. cerevisiae [30]; besides representing an energy source, they also constitute building blocks for lipid membranes [30] and are important for controlling the cellular levels of fatty acids and sterols [31]. Other important yeast lipid classes include sphingolipids, which are involved in the regulation of numerous cellular processes [32]; unsaturated and saturated fatty acids (FA), which are essential constituents of biomembranes [33]; phospholipids [34,35], and sterols, including ergosterol, which contributes to membrane integrity and is reported as the major sterol in S. cerevisiae [36]. The production of volatile compounds by yeasts is influenced by their lipid profile and the availability of lipid sources in the yeast growth medium [37,38], with differences among strains [38] and species [39]. In addition, free FAs are precursors of volatile compounds such as esters, alcohols, and aldehydes [39-41].

Lipidomics is an innovative tool that can be used to understand the biological role of lipids. Despite the complexity of the lipidome of eukaryotic cells, the progress in high throughput techniques based on mass spectrometric approaches allowed the exploration of numerous compound classes. This provides new insight into the molecules and the molecular pathways involved in lipid metabolism. The study of the cellular lipidome involves the description of the functions of lipids and therefore requires knowledge about the molecular basis of the differences in the lipid profiles of certain organelles, the interaction and signaling pathways that involve lipids, and the regulation of the local concentration of lipids in cell compartments [42]. The large number of different existing molecular classes requires complex sample preparation procedures and computational and bioinformatic interpretation of the data, which constitute some of the limitations that may arise in lipidomic studies [43]. Thanks to its relatively simple lipidome and the knowledge of the function and regulation of genes involved in its lipid metabolism, yeasts offer numerous advantages for such studies [44].

Despite the high number of scientific publications focused on lipidomics [35,43,45-48], few studies are available concerning comparative yeast lipidomics [29,49], including publications from the 1970s to the 1990s [34,50,51], and none of these are focused on yeast-insect associations.

In this study, for the first time, the lipidomes of different yeast species associated to SWD were compared. Untargeted lipidomics based on reversed-phase liquid chromatography-quadrupole/ time-of-flight mass spectrometry (RPLC-QTOFMS) was performed on yeast extracts. Six species (H. uvarum, S. cerevisiae, Candida sp., I. terricola, M. pulcherrima and S. vini) were chosen based on previous studies [52]. H. uvarum had a beneficial effect on the survival of SWD larvae [6]. When given as a food source to SWD females, this yeast positively influenced their fecundity; while in capillary feeding assays this species was found to increase ingestion and decrease mortality of SWD adults compared to other yeasts [52]. It was found to be more attractive [19] and to be a preferable food source 
compared to other yeasts [7]. Therefore, three strains (H. u. 1.23, H. u. 2.2 and H. u. 3.4) of this species were included in the study. S. cerevisiae was included as a reference yeast, since detailed information concerning the genome, protein data, and lipidome of this species are available $[29,35,53]$. The other selected species have been already isolated from grapes infested by SWD [6], with H. uvarum being frequently found in association with SWD [10,54] and reported as one of the predominant species $[5,55]$.

The description of the profile of non-polar metabolites of yeasts provides insight about the metabolism of different species cultivated in the same conditions, as well as a list of potential compounds, which may be relevant for the ecology of SWD, and therefore involved in the interaction between microorganisms and SWD.

\section{Results and Discussion}

\subsection{Compound Annotation and Differences in the Lipid Profiles}

The lipidome of the eight selected yeasts was explored. Six cultures of each yeast were included in the dataset to take into account the biological variability. To ensure that all biological replicates were in the same metabolic conditions, all yeast cultures were cultivated under the same growth conditions, and samples were collected at the same time upon reaching the stationary growth phase.

Overall, 171 compounds, including phospholipids (GP), sterols, fatty acids (FA), ceramides (Cer), sphingoid long-chain bases (LCB), monoglycerides (MG), diglycerides (DG) and triglycerides (TG) were annotated. Only known compounds were considered since this study was aimed at finding compounds potentially involved in the interaction between yeasts and SWD. Retention time, ionization mode, annotation level, and mass error (ppm) are reported for each of the annotated compounds in the Supplementary Materials (Table S1). Based on the results of the analysis of variance (ANOVA), significant differences $(p=0.05)$ were found among yeasts concerning all annotated compounds, except for FA(18:0) and DG(16:0_18:1).

A hierarchical clustering dendrogram was generated using Spearman distance and the Ward clustering algorithm to visualize the dataset. The clustering results show that the biological variability is lower in comparison to the differences among species since the species can be divided into subgroups (Figure 1). Two main clusters were observed: cluster one, including C.sp. 3.3, M.p. 3.2, S.v. 1.33 and I.t. 2.1, and cluster two, including the three H. uvarum strains plus S.c. S288c, indicating some similarities in the profiles of lipids in these two groups of species. The three strains belonging to H. uvarum clustered together, indicating a strong similarity between them. Except for S.c. S288c and I.t. 2.1, both of which belong to the family Saccharomycetaceae [56-58], the investigated yeasts belong to different families; therefore, the clustering found does not appear to reflect taxonomic relationships, though specific taxonomic tools and taxonomically defined strains should be considered for classification purposes. This is not surprising, since changes in the lipid metabolism can easily occur [44] and are influenced by the growth conditions and compounds present in the growth medium $[27,28]$, which can supposedly differentially affect the lipid metabolism in the various yeast species. 


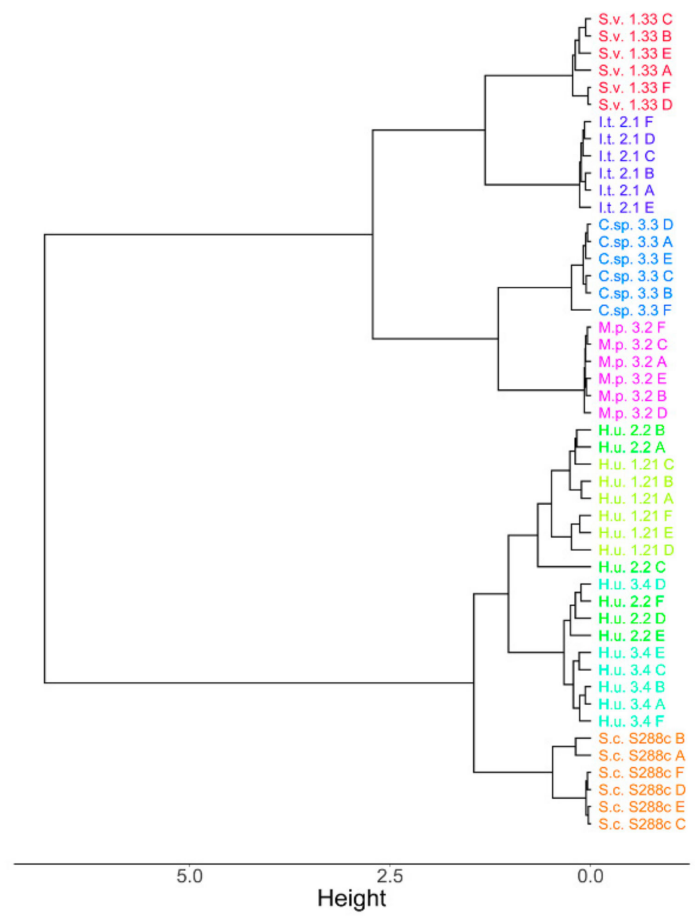

Figure 1. Hierarchical clustering dendrogram obtained using Spearman distance and the Ward clustering algorithm including all the annotated metabolites for each of the six biological replicates (A to F) per yeast.

\subsection{Compound Classes Responsible for Discrimination between Yeast Species}

A principal component analysis (PCA) was performed to highlight the differences between the six yeast species or strains (Figure 2A). Based on the 171 metabolites found, the yeast species could be sorted into discrete clusters. The three strains of H. uvarum clustered together. Augustyn et al. [51] reported an absence of variability among different species in the genus Hanseniaspora based on the cellular FA profile. The results of this study confirm the low variation among strains of this species, also extending the comparison to other lipid classes. The first two principal components explained $64.5 \%$ of the variation in the lipid profile, with principal component one accounting for $43.9 \%$ and principal component two for $20.6 \%$ of the total variation (Figure 2A,B). GPs, DGs, and TGs containing polyunsaturated fatty acids (PUFAs) negatively influenced the first principal component as well as unsaturated free FAs and ergosterol. Principal component two was strongly influenced in a positive direction by most of the FAs, including PUFAs, and negatively by most of the GPs. The statistical significance of the differences among the overall yeast lipidome profiles was determined by pairwise multivariate analysis of variance (MANOVA) of the first five principal components. Principal components four and five were included for the calculation of the MANOVA since they were found to be more informative for the discrimination between $H$. uvarum strains and S. cerevisiae compared to the first three principal components (Figure 2C). Except for H.u. 2.2 and H.u. 1.21 ( $p=0.011)$, H.u. 1.21 and H.u. $3.4(0.026)$, all the species are significantly different from each other $(p=0.005)$ (Figure 2D). 
A

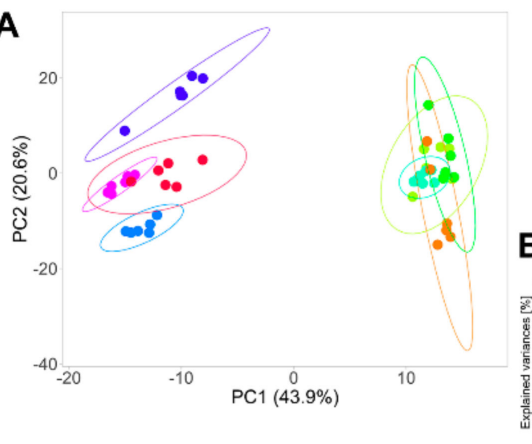

A

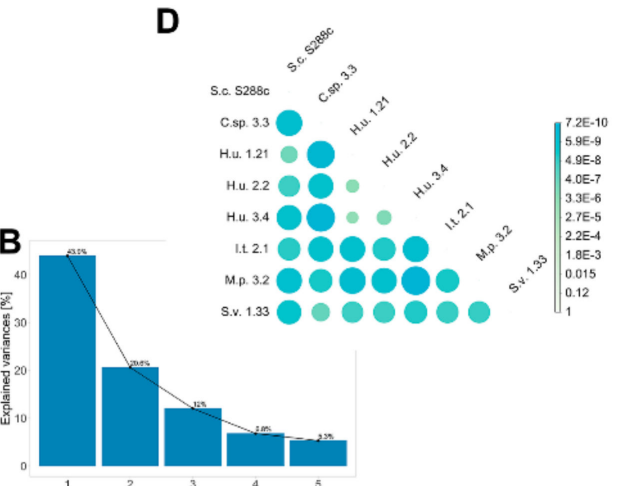

C

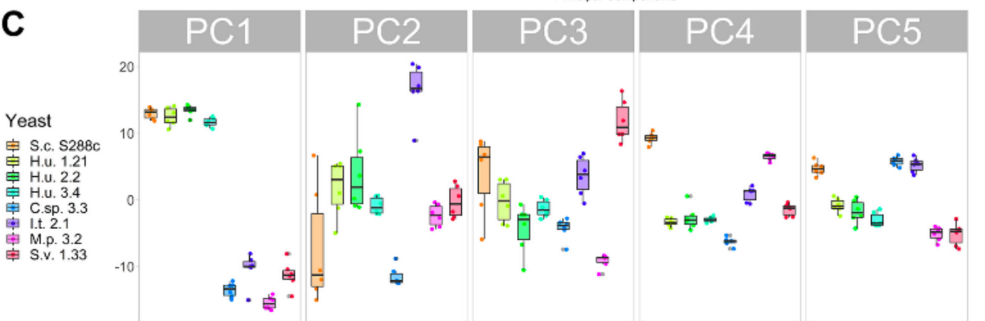

Figure 2. (A) Two-dimensional score plot generated using the first two principal components of the PCA. PCA was performed including all the 171 compounds annotated in six yeasts under investigation. (B) The scree plot shows the variance explained by the first five principal components. (C) Box plots of the scores of each yeast under investigation for the first five principal components. (D) The result of the pairwise MANOVA tests between yeast species based on the first five principal components of the PCA is reported as a heat map indicating significant differences in the yeast lipid chemistry.

\subsection{Differences in the Lipid Profile of GP, DG and TG}

Different classes of phospholipids were found in the analyzed yeast samples, including phosphatidylserine (PS), phosphatidylcholine (PC), phosphatidylethanolamine (PE), phosphatidylglycerol (PG), phosphatidylinositol (PI), lysophosphatidylethanolamine (LPE) and lysophosphatidylcholine (LPC). To visualize the contribution of DGs and TGs as well as the one of GPs to the discrimination between species, heatmaps for different compound classes are shown separately (Figure 3). A similar GP, DG, and TG pattern between the three H. uvarum strains was observed. According to the findings of Hein and Hayen [29], the GP pattern was found to be useful to discriminate between phylogenetically different yeast species. Two species, C.sp. 3.3 and M.p. 3.2, were generally richer in GPs compared to the other analyzed (Figure 3A). In agreement with the findings of Hein and Hayen [29], S.c. S288c was found poor in GPs containing fatty acids with more than two double bonds. Previous studies confirm the lack of PUFAs in S. cerevisiae [49], which is reflected in the presence of only saturated and monounsaturated FAs in the acyl chains of GPs. The same could be observed in the three H. uvarum strains, in accordance with previous studies [51] that reported the absence of C18:2, C18:3, and C16:2 FA both in S. cerevisiae and H. uvarum. TGs were found informative for discrimination between the two species H. uvarum and S. cerevisiae since their profiles are not similar (Figure 3C). Considering DGs and TGs, the main difference between the two big clusters from the hierarchical clustering analysis, namely cluster one including C.sp 3.3, M.p 3.2, I.t. 2.1, S.v. 1.33 and cluster two comprising H. uvarum and S.c. S288c, is the lower amount of compounds containing PUFAs in cluster two. S.v. 1.33 was generally found to possess higher concentrations of a larger variety of DGs and TGs compared to the other yeasts. Interestingly, the presence of odd-numbered fatty acids in the acyl chains of TGs, PCs and LPCs was found in the three species C.sp 3.3, M.p 3.2, and S.v. 1.33. In particular, S.v. 1.33 contained the highest amounts in TGs containing odd-numbered fatty acids, while higher amounts of PCs and LPCs with this characteristic were found in C.sp 3.3. Previous studies indicated that odd-chain fatty acids in yeasts are produced from the elongation of an odd-chain precursor rather than de novo synthesis [59], 
though in some Candida species a small amount of odd-numbered fatty acids was observed even in cells cultivated on even-numbered alkanes [60]. Since microbial lipids mainly contain even-numbered FAs $[61,62]$, this peculiarity constitutes an additional parameter to be considered for discrimination between species.
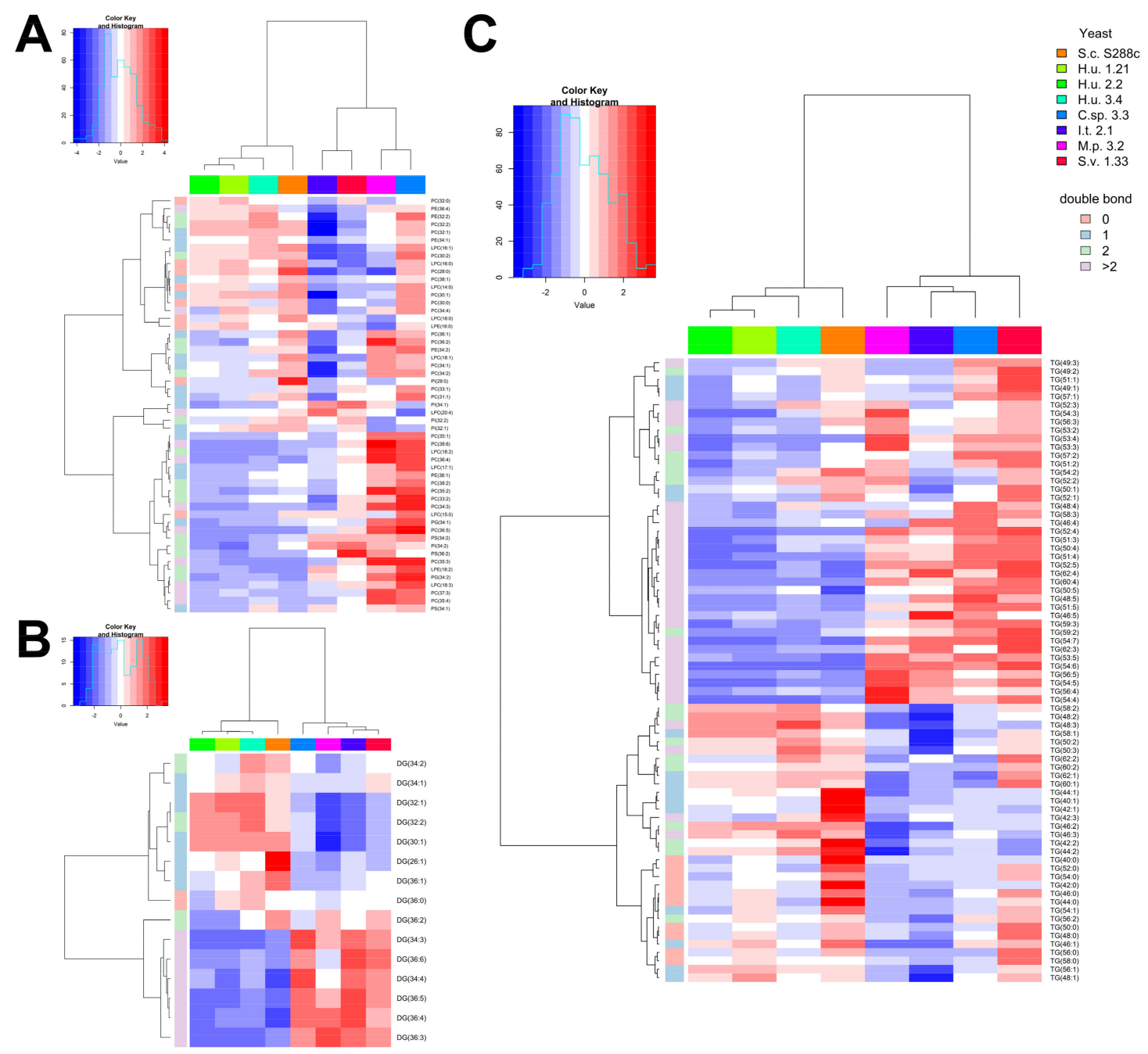

Figure 3. Heatmaps of GPs (A), DGs (B), and TGs (C) in the yeast species and strains included in the study. Intensities of single compounds are displayed using a color scale ranging from red (higher values) to blue (lower values), as shown in the legend. Both rows and columns are clustered using Spearman distance and a Ward clustering algorithm. Average values $(n=6)$ for each yeast are shown.

\subsection{Differences in the Lipid Profile of FA, Ceramides, LCB and Sterols}

Kim et al. [63] showed that the gene Gr64e controls the behavioral as well as the electrophysiological responses of Drosophila to fatty acids, indicating that these compounds are detected by the insect's gustatory system. In capillary feeding assays, Drosophila flies were found to prefer a fatty acid solution rather than water [64]. According to these data, the differences in the chemical compositions of fatty acids among yeast species associated with SWD were of great interest to this study. Oleic acid, palmitoleic acid, palmitic acid, and stearic acid are reported as the major FA of $S$. cerevisiae [27,36,50]. Viljoen et al. [50] also state that the Saccharomycetaceae family, to which $S$. cerevisiae belongs, is characterized by a higher concentration of oleic acid compared to the Saccharomycodaceae, the family to which H. uvarum belongs, and the Metschnikowiaceae, the family of M. pulcherrima. A peculiarity of S. cerevisiae is that it is unable to produce PUFAs with more than two double bonds, while other species can produce unsaturated 
FAs with a variable number of double bonds [65]. More than 20 different FAs were found in the analyzed samples, with differences among species (Table S1). Results confirm the lack of PUFAs in S. cerevisiae (Figure 4), while S.v. 1.33 was the richest species in oleic acid, one of the fatty acids investigated in the above-mentioned studies $[63,64]$. The three H. uvarum strains show, similarly to S. cerevisiae, low amounts of PUFAs (Figure 4). It should be noted that very-long-chain fatty acids with chains longer than 20 carbon atoms were also annotated. These compounds are important lipid components found in all organisms, including S. cerevisiae [66] and, due to the high variability of their concentrations, as well as the complexity of their detection and identification without employing LC-MS techniques [61], only a few studies are dedicated to this group of compounds. Ceramides and long-chain bases (LCB) are constituents of sphingolipids. Four different ceramides and the two LCB phytosphingosine (PHS) and dihydroshphingosine (DHS) were found in the samples analyzed. Considering sterols, only ergosterol was found, with higher relative amounts in S.v. 1.33, I.t. 3.2, and M. p. 3.2 and the lowest amount in H.u. 3.4 (Figure 4).

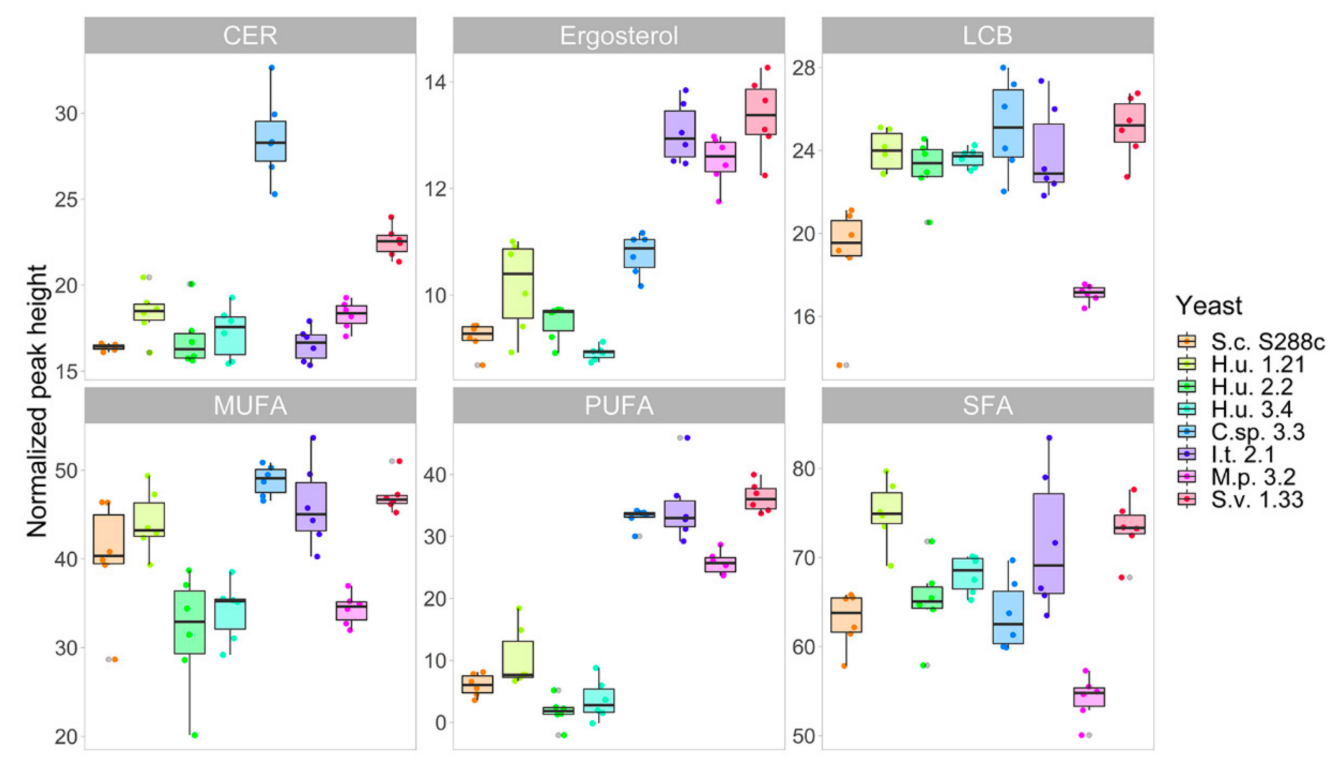

Figure 4. Boxplot of total ceramides (Cer), ergosterol, long-chain base (LCB), monounsaturated fatty acids (MUFA), polyunsaturated fatty acids (PUFA), and saturated fatty acids (SFA) in the eight yeasts included in the study $(n=6)$.

\subsection{Relationship between the Selected Yeasts and SWD}

The variety and diversity of yeast species associated to SWD as well as the wide range of host fruits reflect the insect's ability to adapt to nutrient sources available in the environment [67]. Feeding preferences of Drosophila flies for different yeast-based diets are influenced by numerous factors, including the volatile compounds emitted by yeasts as well as the nutritional composition of the food source $[9,12]$. Yeasts influence several life-history traits of D. melanogaster with different effects on larvae and adult flies [68]. This complex interaction mechanism between yeasts and Drosophila has been widely studied from a behavioral and ecological point of view $[19,25]$ and through the exploration of the volatile compounds involved in attractiveness mechanisms [12,19]. However, little is known about the role of yeast specific metabolites in the association with the insect. In light of previous studies, the results of the present work were evaluated in relation to SWD's dietary preferences and behavior as well as to the effects of different yeast-based diets on SWD flies. As a first approach, the overall profile of the lipid compounds that were annotated was considered. In this case, differences and similarities found between yeast species did not apparently match with SWD preferences or development. For instance, similarities in the global lipid profile between M. pulcherrima and Candida sp. were observed (Figure 2A). In previous studies [6], these two species had different effects on the survival 
of SWD larvae: the two species H. uvarum and Candida sp. had a beneficial effect on the survival of SWD larvae compared to larvae reared on M. pulcherrima. The yeast H. uvarum has been found to be more attractive for SWD flies compared to other Drosophila-associated yeasts, including S. cerevisiae and I. terricola, when offered in a choice test [19]. In addition, Lewis and Hamby [7] demonstrated that larvae of SWD prefer to feed on H. uvarum compared to S. cerevisiae and I. terricola, while Spitaler et al. [52] showed that SWD adults benefit from H. uvarum and S. vini in their diet compared to other species, including S. cerevisiae. In this study, H. uvarum and S. cerevisiae were found to have a similar global lipid profile. Considering the lipid profile in its entirety can be misleading. The fact that SWD flies or larvae do not respond similarly to yeasts that have a generally similar lipid profile does not necessarily indicate that lipids do not have an influence on SWD. Rather, specific compounds or compound classes may play a more relevant role in this mechanism. The information concerning specific compounds is hidden though behind global similarities and diversities. To overcome this problem, another approach was applied for the comparison of the two species H. uvarum and S. cerevisiae. These two yeasts were chosen because, although their lipid profiles were similar, previous studies showed that they give different responses in association with SWD [19]. Additionally, S.c. S288c. is a laboratory strain, while H. uvarum was isolated from grapes infested by SWD flies and is frequently reported in association with SWD [5,55]. A chemical enrichment analysis, an innovative tool for improving the biological and biochemical interpretation of metabolomic data, was performed. ChemRICH is a statistical analysis based on chemical similarities and diversities between groups of metabolites. This approach is normally used for evaluation of metabolic changes like an increase or reduction of the concentrations of specific compound classes that occur moving from one metabolic condition to another [69]. It may be used for the comparison of healthy and unhealthy subjects or treated and control samples. In this study, it was proposed to compare S.c. S288c with H.u. 3.4. Results of the analysis are visualized in a two-dimensional impact plot representing the significantly altered lipid clusters (Figure 5). Enrichment results for each group are reported in Table S2. Lipids belonging to the classes of saturated TG, unsaturated PC, LPC, unsaturated FA, and unsaturated PI were found to have a lower concentration in H.u. 3.4 compared to S.c. S288c (blue nodes), while a higher number of more concentrated saturated FA and unsaturated PE were found in H.u. 3.4 (red nodes). Unsaturated TG, which include the highest number of significantly impacted compounds, and unsaturated DG are characterized by a similar number of lipids having higher or lower concentrations in either of the two yeasts (purple nodes). This approach highlights and categorizes the classes of compounds responsible for these diversities, rather than evidencing the differences based on the global profile of lipids. In this way, it was possible to underline differences between two species, which appear similar based on their global lipid profile. This allows us to focus on specific groups of metabolites that may be considered for further studies like feeding trials and behavioral or survival assays. Spitaler et al. [52] already suggested a possible relationship between the presence of specific polar metabolites, their concentrations, and the survival and feeding stimulation of SWD adults. In this study, a broad qualitative approach was used including a wide range of non-polar compounds. Considering that the same yeast species and microorganisms were grown and collected under the same conditions reported in the paper by Spitaler et al. [52], the results of this work represent an implementation of the above-mentioned study and may support future entomological studies. 


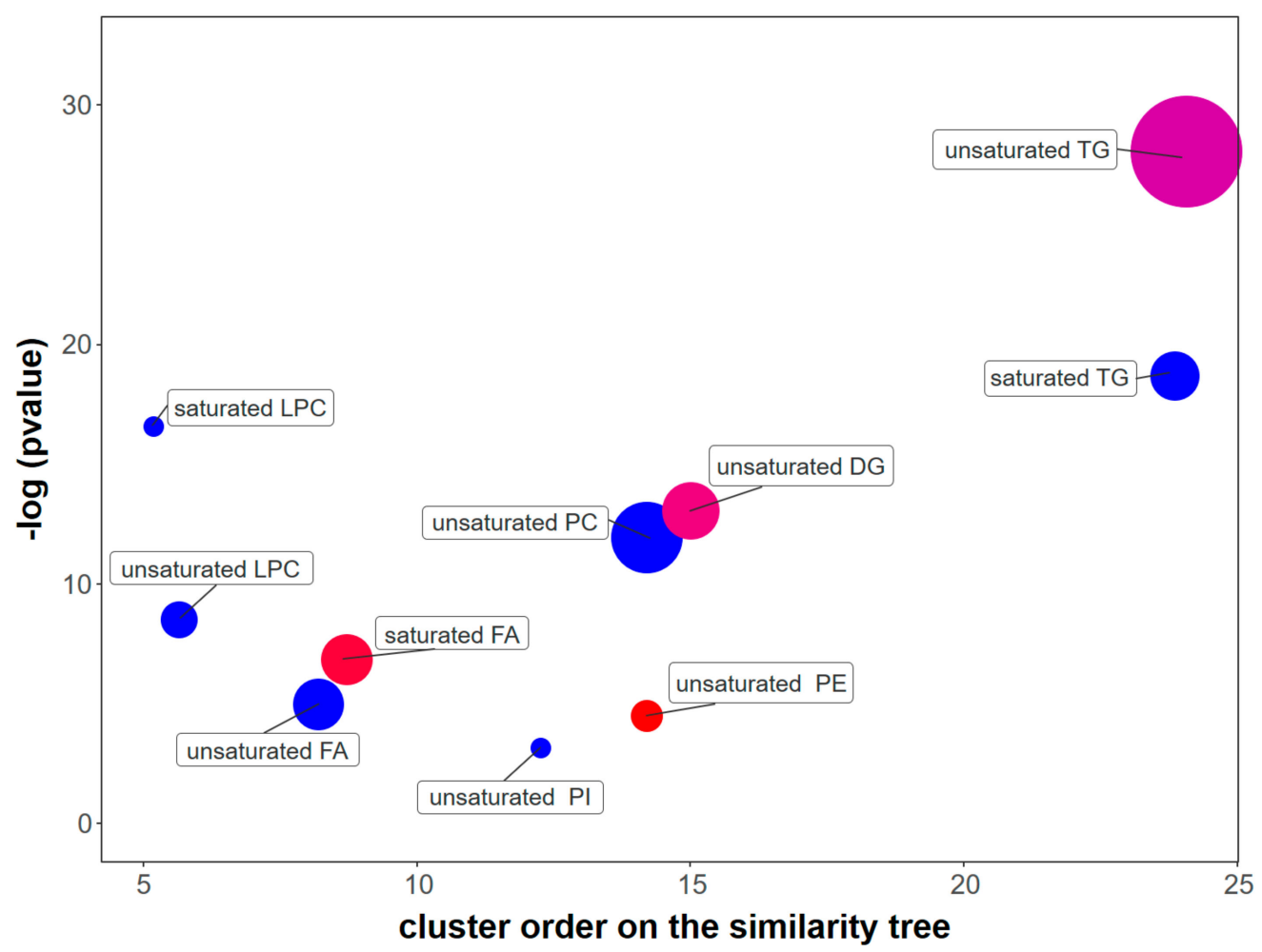

Figure 5. ChemRICH results plot shows the comparison between H.u. 3.4 and S.c. S288c. Each node represents a significantly altered cluster of lipids. The most significantly impacted lipid clusters are at the top of the $y$-axis. Node sizes account for the number of lipids in the clusters, while the node color scale represents the proportion of lipids having higher (red) or lower (blue) concentrations in H.u. 3.4 compared to S.c. S288c. The purple node indicates that in the clusters of unsaturated DG and TG, there is a number of compounds that are more concentrated in H.u. 3.4, and other compounds that are more abundant in S.c. S288c. Only significantly impacted clusters are shown $(p=0.05)$.

\section{Materials and Methods}

\subsection{Chemicals and Growth Media}

Formic acid (LC-MS grade) and MTBE (methyl tertiary butyl ether) (HPLC grade) were obtained from Merck KGaA (Darmstadt, Germany). Acetonitrile (LC-MS grade), isopropanol (LC-MS grade) and methanol (LC-MS grade) were purchased from VWR International Srl (Milan, Italy). Toluene (HPLC grade), ammonium formate (LC-MS grade), ammonium acetate $(\geq 98 \%)$, and analytical internal standards were purchased from Sigma Aldrich (St. Louis, MO, USA). Potato Dextrose Broth (PDB) and Potato Dextrose Agar (PDA) used for yeast cultivation were obtained from Becton, Dickinson and Company (Sparks, MD, USA).

\subsection{Yeasts Cultivation and Lipid Extraction}

Seven yeast cultures with strain numbers containing LB-NB were isolated from feeding channels of SWD larvae in infested grapes in South Tyrol in 2009 [6]. S. cerevisiae strain S288c is a laboratory strain. Yeasts were grown in $220 \mathrm{~mL}$ PDB at $25^{\circ} \mathrm{C}$ for $30 \mathrm{~h}$ in a $250 \mathrm{~mL}$ Erlenmeyer flask closed with cotton and aluminum foil on a rotary shaker at $120 \mathrm{rpm}$. Based on preliminary trials after $30 \mathrm{~h}$, all yeast cultures reached the stationary growth phase. The inoculum $(0.1 \mathrm{~mL})$ was prepared with a loop full of yeast cells cultivated on PDA for four days, which were transferred in a 2-mL Eppendorf tube filled with $1 \mathrm{~mL}$ PDB and vortexed for $10 \mathrm{~s}$ at $1800 \mathrm{rpm}$. Six replicates of the inoculum were prepared for each yeast. The growth of yeasts was evaluated by measuring the optical density at $600 \mathrm{~nm}\left(\mathrm{OD}_{600}\right)$ (Cary 
$60 \mathrm{UV}$-Vis, Agilent) and the yeast cell dry weight (CDW) after $30 \mathrm{~h}$ (centrifugation of fermentation broth, removal of the supernatant, freeze-drying). The list of yeasts included in the study and values of $\mathrm{OD}_{600}$ and $\mathrm{CDW}$ are shown in Table 1.

For the extraction of intracellular lipids, $10 \mathrm{~mL}$ of fermentation broth were quenched in $20 \mathrm{~mL}$ methanol at $-80^{\circ} \mathrm{C}$ and centrifuged (Eppendorf Centrifuge $5810 \mathrm{R}$ ) at $-10^{\circ} \mathrm{C}$ for $5 \mathrm{~min}$ at $4000 \mathrm{rpm}$ using a $-80^{\circ} \mathrm{C}$ prechilled rotor. The supernatant was discarded, and the pellet was freeze-dried. Ten $\mathrm{mg}$ of freeze-dried cell pellet was weighted, and intracellular lipids were extracted using the procedure of Showalter et al. [70]. A volume of $225 \mu \mathrm{L}$ of $-20{ }^{\circ} \mathrm{C}$ methanol containing an internal standard mixture of PE (17:0/17:0), PG (17:0/17:0), sphingosine (d17:1), ceramide (d18:1/17:0), SM (d18:0/17:0), FA (16:0)-d3, PC (12:0/13:0), cholesterol d7, TG d5 (17:0/17:1/17:0), DG (12:0/12:0/0:0), DG (18:1/2:0/0:0), MG (17:0/0:0/0:0), LPC (17:0), LPE (17:1) and $750 \mu \mathrm{L}$ of $-20^{\circ} \mathrm{C}$ MTBE containing the internal standard cholesteryl ester 22:1 was added to the pellet. Samples were shaken for 6 min at $4{ }^{\circ} \mathrm{C}$ using a Thermomixer (Eppendorf) and $188 \mu \mathrm{L}$ of milliQ water was added. Samples were vortexed, centrifuged, and $350 \mu \mathrm{L}$ of the upper layer was collected, evaporated to dryness using a SpeedVac vacuum concentrator, and re-suspended in methanol:toluene $(9: 1, v / v)$ containing $50 \mathrm{ng} \mathrm{mL}^{-1}$ CUDA ((12-[[(cyclohexylamino)carbonyl]amino]-dodecanoic acid). Samples were vortexed, sonicated for $5 \mathrm{~min}$, and centrifuged before analysis. Pooled samples were used as a quality control.

\subsection{Chromatographic and Mass Spectrometric Conditions}

Chromatographic conditions were based on Showalter et al. [70]. A Waters Acquity UPLC CSH C18 (100 mm length $\times 2.1 \mathrm{~mm}$ id; $1.7 \mu \mathrm{m}$ particle size) column with a Waters Acquity VanGuard CSH C18 pre-column $(5 \mathrm{~mm} \times 2.1 \mathrm{~mm}$ id; $1.7 \mu \mathrm{m}$ particle size $)$ maintained at $65{ }^{\circ} \mathrm{C}$ was used for RPLC-QTOFMS analysis. In positive ion mode, solvent A was 60:40 v/v acetonitrile:water with $10 \mathrm{mM}$ ammonium formate and $0.1 \%$ formic acid and solvent $B$ was 90:10 $v / v$ isopropanol:acetonitrile with $10 \mathrm{mM}$ ammonium formate and $0.1 \%$ formic acid. In negative ion mode, solvent $\mathrm{A}$ was $60: 40 \mathrm{v} / \mathrm{v}$ acetonitrile:water with $10 \mathrm{mM}$ ammonium acetate and solvent $\mathrm{B}$ was $90: 10 v / v$ isopropanol:acetonitrile with $10 \mathrm{mM}$ ammonium acetate. The flow rate was set at $0.6 \mathrm{~mL} / \mathrm{min}$, with a 15 min gradient as reported in the paper.

The instrument Impact HD QTOF (Bruker) equipped with an Ultimate 3000 UHPLC (Thermo Scientific, Waltham, MA, USA) was used for LC-MS analysis. Five $\mu \mathrm{L}$ of the re-suspended sample was injected in ESI positive ion mode, while the injection volume in negative ion mode was $10 \mu \mathrm{L}$. The mass spectrometric conditions were as follows: $\mathrm{m} / \mathrm{z}$ range, $60-1700$; capillary voltage, $3500 \mathrm{~V}$; nebulizer gas (nitrogen), $2.4 \mathrm{bar}$; dry gas (nitrogen), $8 \mathrm{~L} / \mathrm{min}$ in positive ion mode and $12 \mathrm{~L} / \mathrm{min}$ in negative ion mode; dry temperature, $325^{\circ} \mathrm{C}$ in positive ion mode and $200{ }^{\circ} \mathrm{C}$ in negative ion mode. For MS/MS, the collision energy was set at $20 \mathrm{eV}$ in positive and in negative ion mode, and the spectra rate was $13 \mathrm{~Hz}$ with 4 precursor ions per cycle. Sodium formate was used as a calibrant for maintaining mass accuracy. 
Table 1. A list of yeasts included in the dataset. Values (Mean \pm SD) of optical density at $600 \mathrm{~nm}\left(\mathrm{OD}_{600}\right)$ and cell dry weight $(\mathrm{CDW})$ of the fermentation broths $(\mathrm{n}=6)$ are reported.

\begin{tabular}{cccccc}
\hline Yeast Species & Strain & Accession Number & Abbreviation & OD $_{\mathbf{6 0 0}}$ & CDW(mg mL $^{-\mathbf{1}}$ Fermentation Broth) \\
\hline Saccharomyces cerevisiae & S288c & - & S.c. S288c & $1.98 \pm 0.03$ & $1.57 \pm 0.18$ \\
Hanseniaspora uvarum & LB-NB-1.21 & KP298009 & H.u. 1.21 & $1.86 \pm 0.02$ & $1.42 \pm 0.11$ \\
Hanseniaspora uvarum & LB-NB-2.2 & MK567898 & H.u. 2.2 & $1.83 \pm 0.05$ & $1.48 \pm 0.15$ \\
Hanseniaspora uvarum & LB-NB-3.4 & MK567905 & H.u. 3.4 & $1.86 \pm 0.04$ & $1.59 \pm 0.06$ \\
Issatchenkia/Picchia terricola & LB-NB-2.1 & MK567903 & I.t. 2.1 & $1.90 \pm 0.04$ & $1.32 \pm 0.17$ \\
Metschnikowia pulcherrima & LB-NB-3.2 & KP298012 & M.p. 3.2 & $2.02 \pm 0.05$ & $1.94 \pm 0.15$ \\
Saccharomycopsis vini & LB-NB-1.33 & KP298011 & S.v. 1.33 & $1.78 \pm 0.10$ & $1.63 \pm 0.13$ \\
Candida sp. & LB-NB-3.3 & KP298013 & C.sp. 3.3 & $2.04 \pm 0.07$ & $1.77 \pm 0.25$ \\
\hline
\end{tabular}

* The accession numbers were deposited in the GenBank NCBI. 


\subsection{Data Processing and Statistics}

For compound identification, full-scan and MS/MS analyses were performed. MS-DIAL was used for deconvolution, peak picking, alignment, and annotation [71]. LipidBlast was used as the library for compound identification with an identification score cut-off of $85 \%$ and a retention time tolerance of $0.1 \mathrm{~min}$. The level of identification of lipids was two for all compounds based on Sumner et al. [72]. The nomenclature used was based on Züllig et al. [73]: the bond type level was reported in the case of annotation based on a high-resolution full-scan (MS1), while double-bond positions were indicated in case of MS/MS-based annotation (Table S1). Peak heights were submitted to Metaboanalyst. Values were normalized using class-based internal standards and further by log transformation before statistical testing. To assess the quality of the data, pooled quality control samples (QCs) were distributed evenly in the analytical batches, RSD\% among the QCs of each internal standard was calculated (Table S3), and the clustering of QCs samples was visually inspected through PCA (Table S3). Pareto scaling was performed for PCA. ANOVA with Tuckey HSD post hoc testing, Student $t$-tests, and pairwise MANOVA tests were done in SPSS (IBM SPSS statistic 24). Graphs were generated using R [74]. To evaluate significantly impacted lipid clusters between S.c. S288c and H.u. 3.4, a chemical similarity enrichment analysis (ChemRICH) was performed using the Kolmogorov-Smirnov-test for statistical analysis [69].

Lipidomics data have been deposited into the EMBL-EBI MetaboLights database [75] with the identifier MTBLS1955. The complete dataset can be accessed here: https:/www.ebi.ac.uk/metabolights/ MTBLS1955.

\section{Conclusions}

The yeast strain has an effect on the attractiveness to SWD flies and affects SWD feeding preferences. Lipids constitute a nutritional source for Drosophila flies; some of these molecules are detected by their gustatory system and are precursors of aromatic compounds. In this study, an untargeted explorative approach was used to investigate the differences in the lipid profile among yeast species naturally occurring in association with SWD. The results constitute a starting point for future investigations on the effects of specific chemical compounds on the behavior of SWD flies. Differences between species were highlighted, as well as the classes of compounds mostly responsible for the discrimination between yeasts. A number of 171 metabolites were annotated. Three strains of H. uvarum under investigation were found to be very similar, and all the other species could be distinguished from each other based on their lipid profiles. ChemRICH enrichment analysis was performed between a laboratory strain (S. cerevisiae) and a species frequently found in association with SWD flies (H. uvarum) to point out diversities within the lipid classes between two species that were reported to differently affect SWD behavior. Significant differences in clusters of lipids were found between the two yeast species. Compounds with significantly higher or lower amounts in either of the two species under investigation belonged to the lipid classes TG, DG, FA, and GP. The information about the differences in the lipid profiles of yeast species associated with SWD may be useful for further entomological and behavioral studies concerning the complex interaction between specific yeasts and insects.

Supplementary Materials: The following are available online at http://www.mdpi.com/2218-1989/10/9/352/s1, Table S1: List of the compounds annotated in the yeasts included in the study (mean $\pm S D, n=6$ ). Peak height values were normalized using class-based internal standards. The internal standards used for the normalization of the peak heights of each compound are reported. The level of identification of lipids for all compounds was two based on Sumner et al. (2007; doi:10.1007/s11306-007-0082-2). Table S2: Results of the ChemRICH analysis of H.u. 3.4 versus S.c. S288c., Table S3: List of the internal standards used. Peak heights found in quality control pooled samples (QCs) and relative standard deviation (RSD\%) are reported for each internal standard. Two-dimensional score plots of all samples, including QCs, generated with all the annotated compounds using the first two principal components of the PCA are reported for the raw dataset and after class-based internal standard normalization and $\log$ transformation. 
Author Contributions: Conceptualization, F.B., U.S., R.F.V., S.S. and D.E.; methodology, F.B. and D.E.; formal analysis, F.B., U.S. and D.E.; investigation, F.B. and D.E.; data curation, F.B. and D.E.; writing-original draft preparation, F.B.; writing-review and editing, U.S., P.R., R.F.V., S.S. and D.E.; visualization, F.B. and D.E.; supervision, P.R., R.F.V., S.S. and D.E.; project administration S.S. and D.E. All authors have read and agreed to the published version of the manuscript.

Funding: This work was supported by the project Dromytal [FESR1021, CUP H32F16000420009] funded by the European Regional Development Fund (ERDF) 2014-2020. Laimburg Research Centre is financed by the Autonomous Province of Bolzano.

Acknowledgments: The authors would like to thank Elke Kaneppele for the assistance with the elaboration of data and Roberto Bianchi for the graphical support. Kathrin Plunger and Cristina Gadotti are gratefully acknowledged for administrative support.

Conflicts of Interest: The authors declare no conflict of interest.

\section{References}

1. Hauser, M. A historic account of the invasion of Drosophila suzukii (Matsumura) (Diptera: Drosophilidae) in the continental United States, with remarks on their identification. Pest Manag. Sci. 2011, 67, 1352-1357. [CrossRef] [PubMed]

2. Cini, A.; Anfora, G.; Escudero-Colomar, L.A.; Grassi, A.; Santosuosso, U.; Seljak, G.; Papini, A. Tracking the invasion of the alien fruit pest Drosophila suzukii in Europe. J. Pest Sci. 2014, 87, 559-566. [CrossRef]

3. De Ros, G.; Conci, S.; Pantezzi, T.; Savini, G. The economic impact of invasive pest Drosophila suzukii on berry production in the Province of Trento, Italy. J. Berry Res. 2015, 5, 89-96. [CrossRef]

4. Dos Santos, L.A.; Mendes, M.F.; Krüger, A.P.; Blauth, M.L.; Gottschalk, M.S.; Garcia, F.R.M. Global potential distribution of Drosophila suzukii (Diptera, Drosophilidae). PLoS ONE 2017, 12, e0174318. [CrossRef]

5. Hamby, K.A.; Hernández, A.; Boundy-Mills, K.; Zalom, F.G. Associations of yeasts with spotted-wing Drosophila (Drosophila suzukii; Diptera: Drosophilidae) in cherries and raspberries. Appl. Environ. Microbiol. 2012, 78, 4869-4873. [CrossRef]

6. Bellutti, N.; Gallmetzer, A.; Innerebner, G.; Schmidt, S.; Zelger, R.; Koschier, E.H. Dietary yeast affects preference and performance in Drosophila suzukii. J. Pest. Sci. 2018, 91, 651-660. [CrossRef] [PubMed]

7. Lewis, M.T.; Hamby, K.A. Differential Impacts of Yeasts on Feeding Behavior and Development in Larval Drosophila suzukii (Diptera: Drosophilidae). Sci. Rep. 2019, 9, 1-12. [CrossRef]

8. Iglesias, L.E.; Nyoike, T.W.; Liburd, O.E. Effect of Trap Design, Bait Type, and Age on Captures of Drosophila suzukii (Diptera: Drosophilidae) in Berry Crops. J. Econ. Entomol. 2014, 107, 1508-1518. [CrossRef]

9. Hamby, K.A.; Becher, P.G. Current knowledge of interactions between Drosophila suzukii and microbes, and their potential utility for pest management. J. Pest. Sci. 2016, 89, 621-630. [CrossRef]

10. Knight, A.L.; Basoalto, E.; Yee, W.; Hilton, R.; Kurtzman, C.P. Adding yeasts with sugar to increase the number of effective insecticide classes to manage Drosophila suzukii (Matsumura) (Diptera: Drosophilidae) in cherry. Pest. Manag. Sci. 2016, 72, 1482-1490. [CrossRef]

11. Mori, B.A.; Whitener, A.B.; Leinweber, Y.; Revadi, S.; Beers, E.H.; Witzgall, P.; Becher, P.G. Enhanced yeast feeding following mating facilitates control of the invasive fruit pest Drosophila suzukii. J. Appl. Ecol. 2017, 54, 170-177. [CrossRef]

12. Becher, P.G.; Flick, G.; Rozpedowska, E.; Schmidt, A.; Hagman, A.; Lebreton, S.; Larsson, M.C.; Hansson, B.S.; Piškur, J.; Witzgall, P.; et al. Yeast, not fruit volatiles mediate Drosophila melanogaster attraction, oviposition and development. Funct. Ecol. 2012, 26, 822-828. [CrossRef]

13. De Camargo, R.; Phaff, H.J. Yeasts occurring in Drosophila flies and in fermenting tomato fruits in Northern California. J. Food Sci. 1957, 22, 367-372. [CrossRef]

14. Carvalho, M.; Sampaio, J.L.; Palm, W.; Brankatschk, M.; Eaton, S.; Shevchenko, A. Effects of diet and development on the Drosophila lipidome. Mol. Syst. Biol. 2012, 8, 1-17. [CrossRef] [PubMed]

15. Yamada, R.; Deshpande, S.A.; Bruce, K.D.; Mak, E.M.; Ja, W.W. Microbes Promote Amino Acid Harvest to Rescue Undernutrition in Drosophila. Cell Rep. 2015, 10, 865-872. [CrossRef] [PubMed]

16. Steck, K.; Walker, S.J.; Itskov, P.M.; Baltazar, C.; Moreira, J.M.; Ribeiro, C. Internal amino acid state modulates yeast taste neurons to support protein homeostasis in Drosophila. Elife 2018, 7, 1-29. [CrossRef]

17. Bing, X.L.; Gerlach, J.; Loeb, G.; Buchon, N. Nutrient-dependent impact of microbes on Drosophila suzukii development. MBio 2018, 9, e02199-17. [CrossRef] 
18. Madden, A.A.; Epps, M.J.; Fukami, T.; Irwin, R.E.; Sheppard, J.; Sorger, D.M.; Dunn, R.R. The ecology of insect-Yeast relationships and its relevance to human industry. Proc. R. Soc. B Biol. Sci. 2018, 285, 20172733. [CrossRef]

19. Scheidler, N.H.; Liu, C.; Hamby, K.A.; Zalom, F.G.; Syed, Z. Volatile codes: Correlation of olfactory signals and reception in Drosophila-yeast chemical communication. Sci. Rep. 2015, 5, 1-13. [CrossRef]

20. Lasa, R.; Navarro-De-La-Fuente, L.; Gschaedler-Mathis, A.C.; Kirchmayr, M.R.; Williams, T. Yeast species, strains, and growth media mediate attraction of Drosophila suzukii (Diptera: Drosophilidae). Insects 2019, 10, 228. [CrossRef]

21. Fanson, B.G.; Taylor, P.W. Protein:carbohydrate ratios explain life span patterns found in Queensland fruit fly on diets varying in yeast:sugar ratios. Age 2012, 34, 1361-1368. [CrossRef]

22. Douglas, A.E. The B vitamin nutrition of insects: The contributions of diet, microbiome and horizontally acquired genes. Curr. Opin. Insect Sci. 2017, 23, 65-69. [CrossRef] [PubMed]

23. Rudkin, G.T.; Schultz, J. Evolution of nutritional requirements in animals; amino-acids essential for Drosophilia melanogaster. Anat. Rec. 1947, 99, 613. [PubMed]

24. Royes, W.V.; Robertson, F.W. The nutritional requirements and growth relations of different species of Drosophila. J. Exp. Zool. 1964, 156, 105-135. [CrossRef]

25. Markow, T.A.; O'Grady, P. Reproductive ecology of Drosophila. Funct. Ecol. 2008, 22, 747-759. [CrossRef]

26. Fahy, E.; Cotter, D.; Sud, M.; Subramaniam, S. Lipid classification, structures and tools. Biochim. Biophys. Acta-Mol. Cell Biol. Lipids 2011, 1811, 637-647. [CrossRef] [PubMed]

27. Tuller, G.; Nemec, T.; Hrastnik, C.; Daum, G. Lipid composition of subcellular membranes of an FY1679-derived haploid yeast wild-type strain grown on different carbon sources. Yeast 1999, 15, 1555-1564. [CrossRef]

28. Tehlivets, O.; Scheuringer, K.; Kohlwein, S.D. Fatty acid synthesis and elongation in yeast. Biochim. Biophys. Acta-Mol. Cell Biol. Lipids 2007, 1771, 255-270. [CrossRef]

29. Hein, E.M.; Hayen, H. Comparative lipidomic profiling of S. cerevisiae and four other hemiascomycetous yeasts. Metabolites 2012, 2, 254-267. [CrossRef]

30. Koch, B.; Schmidt, C.; Daum, G. Storage lipids of yeasts: A survey of nonpolar lipid metabolism in Saccharomyces cerevisiae, Pichia pastoris, and Yarrowia lipolytica. FEMS Microbiol. Rev. 2014, 38, 892-915. [CrossRef]

31. Czabany, T.; Wagner, A.; Zweytick, D.; Lohner, K.; Leitner, E.; Ingolic, E.; Daum, G. Structural and biochemical properties of lipid particles from the yeast Saccharomyces cerevisiae. J. Biol. Chem. 2008, 283, 17065-17074. [CrossRef] [PubMed]

32. Cowart, L.A.; Obeid, L.M. Yeast sphingolipids: Recent developments in understanding biosynthesis, regulation, and function. Biochim. Biophys. Acta Mol. Cell Biol. Lipids 2007, 1771, 421-431. [CrossRef] [PubMed]

33. Pina, C.; Santos, C.; Couto, J.A.; Hogg, T. Ethanol tolerance of five non-Saccharomyces wine yeasts in comparison with a strain of Saccharomyces cerevisiae-Influence of different culture conditions. Food Microbiol. 2004, 21, 439-447. [CrossRef]

34. Kaneko, H.; Hosohara, M.; Tanaka, M.; Itoh, T. Lipid composition of 30 species of yeast. Lipids 1976, 11, 837-844. [CrossRef] [PubMed]

35. Ejsing, C.S.; Sampaio, J.L.; Surendranath, V.; Duchoslav, E.; Ekroos, K.; Klemm, R.W.; Simons, K.; Shevchenko, A. Global analysis of the yeast lipidome by quantitative shotgun mass spectrometry. Proc. Natl. Acad. Sci. USA 2009, 106, 2136-2141. [CrossRef]

36. Klug, L.; Daum, G. Yeast lipid metabolism at a glance. FEMS Yeast Res. 2014, 14, 369-388. [CrossRef]

37. Pires, E.J.; Teixeira, J.A.; Brányik, T.; Vicente, A.A. Yeast: The soul of beer's aroma-A review of flavour-active esters and higher alcohols produced by the brewing yeast. Appl. Microbiol. Biotechnol. 2014, 98, 1937-1949. [CrossRef]

38. Rollero, S.; Mouret, J.R.; Sanchez, I.; Camarasa, C.; Ortiz-Julien, A.; Sablayrolles, J.M.; Dequin, S. Key role of lipid management in nitrogen and aroma metabolism in an evolved wine yeast strain. Microb. Cell Fact. 2016, 15, 1-15. [CrossRef]

39. Aslankoohi, E.; Herrera-Malaver, B.; Rezaei, M.N.; Steensels, J.; Courtin, C.M.; Verstrepen, K.J. Non-conventional yeast strains increase the aroma complexity of bread. PLoS ONE 2016, 11, e0165126. [CrossRef] 
40. Fellman, J.K.; Miller, T.W.; Mattinson, D.S.; Mattheis, J.P. Factors that influence biosynthesis of volatile flavor compounds in apple fruits. HortScience 2000, 35, 1026-1033. [CrossRef]

41. Song, J.; Bangerth, F. Fatty acids as precursors for aroma volatile biosynthesis in pre-climacteric and climacteric apple fruit. Postharvest Biol. Technol. 2003, 30, 113-121. [CrossRef]

42. Van Meer, G. Cellular lipidomics. EMBO J. 2005, 24, 3159-3165. [CrossRef] [PubMed]

43. Wenk, M.R. The emerging field of lipidomics. Nat. Rev. Drug Discov. 2005, 4, 594-610. [CrossRef]

44. Gaspar, M.L.; Aregullin, M.A.; Jesch, S.A.; Nunez, L.R.; Villa-García, M.; Henry, S.A. The emergence of yeast lipidomics. Biochim. Biophys. Acta Mol. Cell Biol. Lipids 2007, 1771, 241-254. [CrossRef] [PubMed]

45. Watson, A.D. Lipidomics: A global approach to lipid analysis in biological systems. J. Lipid Res. 2006, 47, 2101. [CrossRef] [PubMed]

46. Wolf, C.; Quinn, P.J. Lipidomics: Practical aspects and applications. Prog. Lipid Res. 2008, 47, 15-36. [CrossRef]

47. Li, M.; Yang, L.; Bai, Y.; Liu, H. Analytical methods in lipidomics and their applications. Anal. Chem. 2014, 86, 161-175. [CrossRef]

48. Casanovas, A.; Sprenger, R.R.; Tarasov, K.; Ruckerbauer, D.E.; Hannibal-Bach, H.K.; Zanghellini, J.; Jensen, O.N.; Ejsing, C.S. Quantitative analysis of proteome and lipidome dynamics reveals functional regulation of global lipid metabolism. Chem. Biol. 2015, 22, 412-425. [CrossRef]

49. Aloklah, B.; Alhajali, A.; Yaziji, S. Identification of some yeasts by fatty acid profiles. Polish J. Microbiol. 2014, 63, 467-472. [CrossRef]

50. Viljoen, B.C.; Kock, J.L.F.; Lategan, P.M. Fatty acid composition as a guide to the classification of selected genera of yeasts belonging to the endomycetales. J. Gen. Microbiol. 1986, 132, 2397-2400. [CrossRef]

51. Augustyn, O.P.H.; Ferreira, D.; Kock, J.L.F. Differentiation between Yeast Species, and Strains within a Species, by Cellular Fatty Acid Analysis: 4. Saccharomyces sensu stricto, Hanseniaspora, Saccharomycodes and Wickerhamiella. Syst. Appl. Microbiol. 1991, 14, 324-334. [CrossRef]

52. Spitaler, U.; Bianchi, F.; Eisenstecken, D.; Castellan, I.; Angeli, S.; Dordevic, N.; Robatscher, P.; Vogel, R.F.; Koschier, E.H.; Schmidt, S. Yeast species affects feeding and fitness of Drosophila suzukii adults. J. Pest Sci. 2020, 93, 1295-1309. [CrossRef]

53. Jewison, T.; Knox, C.; Neveu, V.; Djoumbou, Y.; Guo, A.C.; Lee, J.; Liu, P.; Mandal, R.; Krishnamurthy, R.; Sinelnikov, I.; et al. YMDB: The yeast metabolome database. Nucleic Acids Res. 2012, 40, D815-D820. [CrossRef] [PubMed]

54. Chandler, J.A.; Eisen, J.A.; Kopp, A. Yeast communities of diverse Drosophila species: Comparison of two symbiont groups in the same hosts. Appl. Environ. Microbiol. 2012, 87, 559-566. [CrossRef] [PubMed]

55. Fountain, M.T.; Bennett, J.; Cobo-Medina, M.; Conde Ruiz, R.; Deakin, G.; Delgado, A.; Harrison, R.; Harrison, N. Alimentary microbes of winter-form Drosophila suzukii. Insect Mol. Biol. 2018, 27, 383-392. [CrossRef] [PubMed]

56. Crous, P.W.; Gams, W.; Stalpers, J.A.; Robert, V.; Stegehuis, G. MycoBank: An online initiative to launch mycology into the 21st century. Stud. Mycol. 2004, 50, 19-22.

57. Robert, V.; Stegehuis, G.; Stalpers, J. Mycobank. The MycoBank Engine and Related Databases 2005. Available online: http://www.mycobank.org/defaultinfo.aspx?Page=Home (accessed on 30 January 2020).

58. Robert, V.; Vu, D.; Amor, A.B.H.; van de Wiele, N.; Brouwer, C.; Jabas, B.; Szoke, S.; Dridi, A.; Triki, M.; Daoud, S.B.; et al. MycoBank gearing up for new horizons. IMA Fungus 2013, 4, 371-379. [CrossRef]

59. Rattray, J.B.M.; Schibeci, A.; Kidby, D.K. Lipids of yeasts. Bacteriol. Rev. 1975, 39, 197-231. [CrossRef]

60. Mishina, M.; Yanagawa, S.; Tanaka, A.; Fukui, S. Effects of chain-length of alkane substrate on fatty acid composition and biosynthetic pathway in some Candida yeasts. Agric. Biol. Chem. 1973, 37, 863-870. [CrossRef]

61. Ǩezanka, T.; Sigler, K. Odd-numbered very-long-chain fatty acids from the microbial, animal and plant kingdoms. Prog. Lipid Res. 2009, 48, 206-238. [CrossRef]

62. Kondo, N.; Ohno, Y.; Yamagata, M.; Obara, T.; Seki, N.; Kitamura, T.; Naganuma, T.; Kihara, A. Identification of the phytosphingosine metabolic pathway leading to odd-numbered fatty acids. Nat. Commun. 2014, 5, 5338. [CrossRef] [PubMed]

63. Kim, H.; Kim, H.; Kwon, J.Y.; Seo, J.T.; Shin, D.M.; Moon, S.J. Drosophila Gr64e mediates fatty acid sensing via the phospholipase C pathway. PLoS Genet. 2018, 14, e1007229. [CrossRef] [PubMed] 
64. Masek, P.; Keene, A.C. Drosophila Fatty Acid Taste Signals through the PLC Pathway in Sugar-Sensing Neurons. PLoS Genet. 2013, 9, e1003710. [CrossRef] [PubMed]

65. Yazawa, H.; Iwahashi, H.; Kamisaka, Y.; Kimura, K.; Uemura, H. Production of polyunsaturated fatty acids in yeast Saccharomyces cerevisiae and its relation to alkaline $\mathrm{pH}$ tolerance. Yeast 2009, 26, 167-184. [CrossRef]

66. Rezanka, T. Very-long-chain fatty acids from the animal and plant kingdoms. Prog. Lipid Res. 1989, 28, 147-187. [CrossRef]

67. Silva-Soares, N.F.; Nogueira-Alves, A.; Beldade, P.; Mirth, C.K. Adaptation to new nutritional environments: Larval performance, foraging decisions, and adult oviposition choices in Drosophila suzukii. BMC Ecol. 2017, 17, 21. [CrossRef]

68. Anagnostou, C.; Dorsch, M.; Rohlfs, M. Influence of dietary yeasts on Drosophila melanogaster life-history traits. Entomol. Exp. Appl. 2010, 136,1-11. [CrossRef]

69. Barupal, D.K.; Fiehn, O. Chemical Similarity Enrichment Analysis (ChemRICH) as alternative to biochemical pathway mapping for metabolomic datasets. Sci. Rep. 2017, 7, 14567. [CrossRef]

70. Showalter, M.R.; Nonnecke, E.B.; Linderholm, A.L.; Cajka, T.; Sa, M.R.; Lönnerdal, B.; Kenyon, N.J.; Fiehn, O. Obesogenic diets alter metabolism in mice. PLoS ONE 2018, 13, e0190632. [CrossRef]

71. Tsugawa, H.; Cajka, T.; Kind, T.; Ma, Y.; Higgins, B.; Ikeda, K.; Kanazawa, M.; Vandergheynst, J.; Fiehn, O.; Arita, M. MS-DIAL: Data-independent MS/MS deconvolution for comprehensive metabolome analysis. Nat. Methods 2015, 12, 523-526. [CrossRef]

72. Sumner, L.W.; Amberg, A.; Barrett, D.; Beale, M.H.; Beger, R.; Daykin, C.A.; Fan, T.W.M.; Fiehn, O.; Goodacre, R.; Griffin, J.L.; et al. Proposed minimum reporting standards for chemical analysis. Metabolomics 2007, 3, 211-221. [CrossRef] [PubMed]

73. Züllig, T.; Trötzmüller, M.; Köfeler, H.C. Lipidomics from sample preparation to data analysis: A primer. Anal. Bioanal. Chem. 2020, 412, 2191-2209. [CrossRef] [PubMed]

74. Team, R.C. R: A Language and Environment for Statistical Computing; R Foundation for Statistical Computing: Vienna, Austria, 2019.

75. Haug, K.; Cochrane, K.; Nainala, V.C.; Williams, M.; Chang, J.; Jayaseelan, K.V.; O’Donovan, C. MetaboLights: A resource evolving in response to the needs of its scientific community. Nucleic Acids Res. 2020, 48, D440-D444. [CrossRef] [PubMed] 